\title{
SIMULAÇÃO DA PRODUÇÃO DE HIDROGÊNIO APLICANDO FLUIDODINÂMICA COMPUTACIONAL
}

\author{
SANTOS, D. da C. ${ }^{1}$ JESUS, L. F. S. ${ }^{1}$ NETTO, L. D. de S. ${ }^{1}$ FERRAZ, C. ${ }^{1}$ JESUS, E. ${ }^{1}$ SILVA, \\ A. S. ${ }^{2}$ PAGANO, R.L. ${ }^{1}$ \\ ${ }^{1}$ Universidade Federal de Sergipe / Departamento de Engenharia Química \\ 2 Universidade Federal de Sergipe / Departamento de Matemática \\ e-mail para contato: rpagano@ufs.br
}

\begin{abstract}
RESUMO - O hidrogênio tem sua principal produção a partir da reação reforma a vapor. Devido às características desta reação, para se alcançar conversões razoáveis são necessárias altas temperaturas e pressões em reatores convencionais. A literatura apresenta propostas de utilização de reatores com membrana com o intuito de deslocar o equilíbrio termodinâmico, favorecendo maiores conversões, em menores temperaturas operacionais. Neste cenário, este trabalho propôs a simulação de um reator de membrana para produção de hidrogênio a partir da reação de reforma a vapor aplicando o software ANSYS CFX ${ }^{\circledR}$. Inicialmente, a geometria do reator utilizando o software geometry foi elaborada e um estudo de convergência de malha foi realizado. Por fim, o reator foi simulado com diferentes condições operacionais e seus resultados corroboram os encontrados na literatura.
\end{abstract}

\section{INTRODUÇÃO}

Em virtude da necessidade mundial por fontes de energia renováveis, das mudanças climáticas causadas pelo aquecimento global, da diminuição e, possivelmente, extinção das reservas de combustíveis fósseis, torna-se imprescindível a pesquisa e busca por novas alternativas e processos geradores de energia. Diante disso, a produção de hidrogênio aparece como uma alternativa válida, sendo uma fonte renovável e menos agressora ao meio ambiente. Muitos estudos e tecnologias estão sendo desenvolvidos para a produção do hidrogênio. Industrialmente, a principal forma de obtenção é através da reforma catalítica a vapor do gás natural. Segundo Sá (2004), a reforma a vapor é um tipo de tecnologia largamente utilizada por possuir uma melhor razão $\mathrm{H}_{2} / \mathrm{C}$. Este processo sob condições reacionais adequadas, leva à formação do gás de síntese formado por gás hidrogênio e monóxido de carbono (Besteti et al., 2005).

A utilização de reatores de membrana é um grande avanço nesta produção. Sendo que tanto a reação de produção quanto a separação do hidrogênio do meio reacional ocorrem simultaneamente em um único processo. Este fato proporciona vantagens como: redução do volume ocupado para produção do hidrogênio e aumento da conversão dos reagentes (Smith et al., 2011). A utilização destes reatores é bastante útil na reação de obtenção de hidrogênio através da reforma a vapor. 


\section{9 a 22 de outubro de 2014 \\ Florianópolis/SC}

A modelagem matemática e a simulação computacional aplicando fluidodinâmica computacional (CFD) torna-se peça fundamental de investigação, permitindo descrever os fenômenos e determinar as melhores condições operacionais do processo, detalhando seu comportamento e apresentando uma solução condizente com a realidade, sem que haja perda de matéria prima (Besteti et al, 2005).

Este trabalho teve como finalidade simular um reator de membrana em escala laboratorial com membrana de paládio. O modelo matemático foi desenvolvido com base nas configurações geométricas e operações do reator apresentado por Shu et al. (1994). O software ANSYS CFX 14.0 foi utilizado para realizar as simulações do processo apresentado. Os dados obtidos foram comparados com os fornecidos na literatura, avaliando assim a eficiência do processo simulado.

\section{REVISÃO BIBLIOGRÁFICA}

\subsection{Produção de Hidrogênio}

O hidrogênio é considerado um combustível de queima limpa, contudo esta denominação dependerá da matéria-prima e da fonte de energia utilizada para o processo de obtenção. A produção de hidrogênio pode ocorrer por diversos processos: oxidação parcial, reforma autotérmica, eletrólise da água, processos biológicos e fotobiológicos por enzimas dentre outros (Souza et al., 2004), entretanto, a rota de produção mais estudada é a da reforma a vapor. As reações envolvidas na reforma a vapor compreendem as reações de conversão do metano em monóxido de carbono e hidrogênio e a reação de deslocamento gás-água, como mostram as equações a seguir.

$$
\begin{array}{ll}
\mathrm{CH}_{4}+\mathrm{H}_{2} \mathrm{O} \leftrightarrow \mathrm{CO}+3 \mathrm{H}_{2} & \Delta \mathbf{H}_{298 \mathrm{~K}}^{\circ}=206,1 \mathrm{~kJ} \cdot \mathrm{mol}^{-1} \\
\mathrm{CO}+\mathrm{H}_{2} \mathrm{O} \leftrightarrow \mathrm{CO}_{2}+\mathrm{H}_{2} & \Delta \mathbf{H}_{298 \mathrm{~K}}^{\circ}=-41,15 \mathrm{~kJ} \cdot \mathrm{mol}^{-1} \\
\mathrm{CH}_{4}+2 \mathrm{H}_{2} \mathrm{O} \leftrightarrow \mathrm{CO}_{2}+4 \mathrm{H}_{2} & \mathbf{\Delta H}_{298 \mathrm{~K}}^{\circ}=165,0 \mathrm{~kJ} \cdot \mathrm{mol}^{-1}
\end{array}
$$

As reações da reforma a vapor têm um mecanismo global endotérmico, requerendo um controle de temperatura de modo a manter favoráveis as condições de equilíbrio químico. Visando este controle, a literatura vem propondo a utilização de reatores com membranas que permitam a percolação seletiva do hidrogênio. A reação para a formação de produtos é favorecida, permitindo que esta seja realizada sob temperaturas moderadas, alcançando altas conversões (Mùnera et al., 2003).

\subsection{Reatores com Membrana}

Os reatores convencionais que possuem membranas são dispositivos compostos basicamente por um reator e uma membrana. Contudo, a membrana pode estar fisicamente integrada ao reator ou ser um dispositivo localizado externamente ao mesmo. Os principais objetivos da utilização de membranas é deslocar o equilíbrio termodinâmico, aumentando assim a conversão, o rendimento do produto, e reprimir reações secundárias indesejadas do produto. De acordo com Bernardes (2009), os reatores com membrana quando utilizados em reações catalíticas, a membrana pode ter ou não função 
de um catalisador. $\mathrm{O}$ reator com membrana catalítica pode ter tanto a membrana atuando como único catalisador, ou possuir um catalisador convencional além da membrana. O esquema geral de um reator de membrana pode ser visualizado na Figura 1.

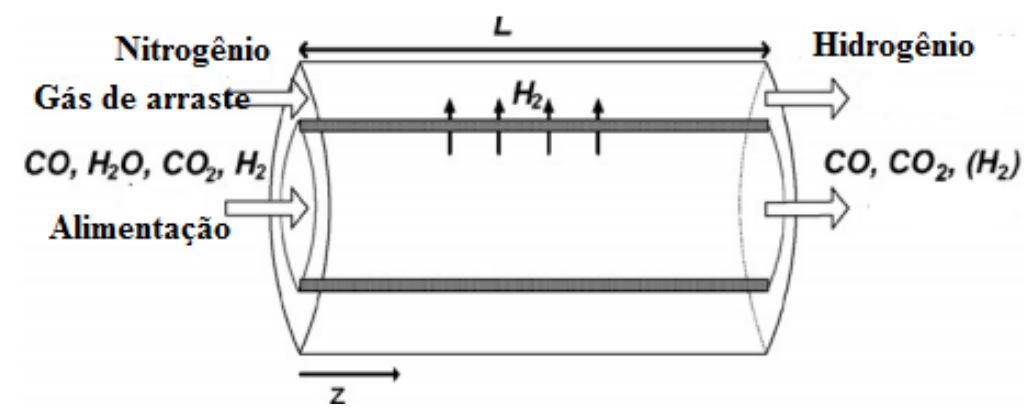

Figura 1: Esquema de um reator de membrana.

Diante das vantagens apresentadas, os reatores com membrana vem sendo aplicados nas mais diversas áreas, tais como, petroquímica, farmacêutica, metalúrgica, tratamento de águas e produção de hidrogênio, justificando o seu destaque nas pesquisas durante as últimas décadas.

\subsection{Fluidodinâmica Computacional}

A Fluidodinâmica Computacional (Computational Fluid Dynamics - CFD) é uma ferramenta que simula o comportamento de sistemas envolvendo fenômenos de transporte, transferência de calor e de massa, dentre outros processos físico/físico-químicos relacionados. Esta ferramenta aborda a descrição numérica do escoamento e, apesar das suas incontáveis vantagens, os seus resultados são válidos apenas enquanto os modelos físicos incorporados nas equações e nas condições de contorno forem válidos. Portanto, os resultados estão sujeitos a erros, principalmente em se tratando de escoamentos mais complexos, como os turbulentos (Andrade, 2013).

\section{METODOLOGIA}

A modelagem do reator de membrana foi realizada supondo o fluxo em estado estacionário tridimensional para as equações de massa, energia e movimento, regime laminar em condições nãoisotérmicas, tais equações estão apresentadas na Tabela 1.

A cinética de reação adotada no presente trabalho foi a apresentada por Froment e Xu (1989) para descrever a reação da reforma a vapor do metano, a mesma considera o equilíbrio termodinâmico da reação e parâmetros de adsorção das espécies envolvidas. As taxas de reações contidas na Tabela 2 descrevem as reações mostradas nas Equações (1), (2) e (3), sendo que ki, Ki, pj e Kj representam os coeficientes da taxa de reação, as constantes de equilíbrio da reação, a pressão parcial do componente $j$ e a constante de adsorção do componente $j$, respectivamente. Ainda, têm-se que o subscrito $i$ indica a reação com valores 1,2 e 3 e $j$ corresponde aos componentes, ou seja, $\mathrm{CO}, \mathrm{CO}_{2}, \mathrm{H}_{2} \mathrm{O}, \mathrm{CH}_{4}$ e $\mathrm{H}_{2}$. Além disso, a fase gasosa foi modelada considerando a equação de estado de Soave-Redlich-Kwong. 
Tabela 1: Equações de balanço de massa, energia e movimento.

\begin{tabular}{lc}
\hline Massa & Movimento \\
\hline & $\begin{array}{r}\frac{\partial}{\partial t}(\rho \vec{v})+\nabla \cdot(\rho \vec{v} \vec{v}) \\
=-\nabla p+\nabla:(\overline{\bar{\tau}})+\rho \vec{g}+\vec{F}\end{array}$ \\
\hline Energia & \\
$\qquad \frac{\partial}{\partial t}(\rho E)+\nabla \cdot(\overrightarrow{\boldsymbol{v}}(\rho E+p))=\nabla \cdot\left(k_{e f f} \nabla T-\sum_{j} h_{j} \vec{J}_{j}+\left(\overline{\bar{\tau}}_{e f f} \cdot \overrightarrow{\boldsymbol{v}}\right)\right)+S_{h}$
\end{tabular}

Tabela 2: Cinética química para a reação de reforma a vapor do metano.

$$
\begin{gathered}
r_{1}=\frac{\frac{k_{1}}{p_{H_{2}}^{2,5}}\left(p_{C H_{4}} p_{H_{2} O}-\frac{p_{H_{2}}^{3} p_{C O}}{K_{1}}\right)}{(D E N)^{2}} \\
r_{3}=\frac{\frac{k_{3}}{p_{H_{2}}^{3,5}\left(p_{C H_{4}} p_{H_{2} O}^{2}-\frac{p_{H_{2}}^{4} p_{C O}}{K_{3}}\right)}}{(D E N)^{2}} \\
D E N=1+K_{C O} p_{C O}+K_{H_{2}} p_{H_{2}}+K_{C H_{4}} p_{C H_{4}}+\frac{K_{H_{2} O} p_{H_{2} O}}{p_{H_{2}}}
\end{gathered}
$$

A simulação do reator de membrana para produção de hidrogênio foi realizada utilizando o pacote comercial de Fluidodinâmica Computacional da ANSYS CFX 14.0. O reator foi projetado conforme as características geométricas e condições contorno mostradas na Tabela 3, adaptadas de Shu et al. (1994). A cinética da reação foi incluída no modelo matemático como um termo de geração de massa, termo fonte $S_{\mathrm{m}}$.

Para avaliar a influência da malha nas simulações desenvolvidas para o reator de membrana, um estudo de convergência de malha foi inicialmente realizado. A metodologia utilizada para o estudo de convergência para os diferentes refinos das malhas pode ser resumida nos seguintes passos de acordo com a American Society of Mechanical Engineers (ASME, 2008): (i) Definir uma célula representativa para cálculos tridimensionais a partir da Equação (4), sendo $V_{i}$ o volume de cada elemento da célula e $N$ o número total de células usadas para os cálculos; (ii) Selecionar diferentes refinos de malha, sendo que é aconselhável que a razão entre os refinos $r=h_{\text {grosseiro }} / h_{\text {refinado }}$ seja maior que 1,3, valor este baseado em estudos empíricos; (iii) A sequência de refino deve ser 
organizada numa ordem em que $h_{1}<h_{2}<h_{3}<h_{4}<h_{5}$. Em seguida, os parâmetros de ordem $p, q$ e $s$ devem ser calculados utilizando as Equações $5 \mathrm{a}$, $5 \mathrm{~b}$ e $5 \mathrm{c}$, sendo $e_{32}=\phi_{3}-\phi_{2}$ e $e_{32}=\phi_{2}-\phi_{1}$ $\operatorname{com} \phi$ sendo um ponto no volume de controle no qual ocorre uma alteração da variável sob análise; (iv) Calcular os valores extrapolados a partir da Equação (6) e de forma similar calcular o valor de $\phi_{\text {ext }}^{32}$; (v) Calcular a estimativa de erro para cada alteração de refino da malha a partir dos seguintes parâmetros: Erro aproximado, Equação (7); Erro extrapolado, Equação (8) e Índice de convergência da malha, Equação (9).

Tabela 3: Características geométricas para construção da geometria do reator de membrana

\begin{tabular}{|c|c|c|}
\hline Temperatura na região reacional (Tr) & $793,15 \mathrm{~K}$ & \\
\hline Pressão de entrada na região reacional & 29 bar & \\
\hline Temperatura na região anular (Ta) & $1043 \mathrm{~K}$ & \\
\hline Pressão de entrada na região reacional & 1 bar & \\
\hline Comprimento do reator $(\mathrm{L})$ & $12 \mathrm{~m}$ & \\
\hline Diâmetro interno do reator tubular & $0,1016 \mathrm{~m}$ & \\
\hline Diâmetro externo do reator tubular & $0,1322 \mathrm{~m}$ & \\
\hline Espessura da membrana de paládio & $20 \cdot 10^{-6} \mathrm{~m}$ & \\
\hline Permeabilidade da membrana & $2,19 \cdot 10^{-5} \exp \left(\frac{-29730 J}{8,314 . T}\right)$ & $\frac{m o l}{m \cdot s \cdot P a^{0,5}}$ \\
\hline $\begin{array}{l}\text { Densidade do leito catalítico } \\
\text { Alimentação na entrada: }\end{array}$ & $2270 \mathrm{~kg} / \mathrm{m}^{3}$ & \\
\hline $\mathrm{CH}_{4}$ & $5,168 \mathrm{kmol} / \mathrm{h}$ & \\
\hline $\mathrm{H}_{2} \mathrm{O}$ & $17,354 \mathrm{kmol} / \mathrm{h}$ & \\
\hline $\mathrm{CO}_{2}$ & $0,289 \mathrm{kmol} / \mathrm{h}$ & \\
\hline $\mathrm{H}_{2}$ & $0,630 \mathrm{kmol} / \mathrm{h}$ & \\
\hline $\mathrm{N}_{2}$ & $0,848 \mathrm{kmol} / \mathrm{h}$ & \\
\hline
\end{tabular}

No trabalho em questão foi analisado interferência do refino da malha na temperatura, logo, o $\phi$ representará os valores de temperatura no reator de membrana em uma posição definida.

$$
\begin{aligned}
& h=\left[\frac{1}{N} \sum_{i=1}^{N}\left(V_{i}\right)\right]^{\frac{1}{3}} \\
& p=\frac{1}{\ln \left(r_{21}\right)}|\ln | e_{32} / e_{21}|+q(p)| \\
& q(p)=\ln \left(\frac{r_{21}^{p}-s}{r_{32}^{p}-s}\right) \\
& s=1 . \operatorname{sgn}\left(\frac{e_{32}}{e_{21}}\right) \\
& \phi_{\text {ext }}^{21}=\left(r_{21}^{p} \phi_{1}-\phi_{2}\right) /\left(r_{21}^{p}-1\right)
\end{aligned}
$$




$$
\begin{aligned}
& e_{a}^{21}=\left|\frac{\phi_{1}-\phi_{2}}{\phi_{1}}\right| \\
& e_{\text {ext }}^{21}=\left|\frac{\phi_{\text {ext }}^{21}-\phi_{1}}{\phi_{\text {ext }}^{21}}\right| \\
& G C I_{21}=\frac{1,25 \cdot e_{a}^{21}}{r_{21}^{p}-1}
\end{aligned}
$$

\section{RESULTADOS E DISCUSSÃO}

A metodologia proposta pela ASME (2008) foi utilizada para construir os diferentes refinos de malha para o reator de membrana, considerando a temperatura como variável a ser monitorada no estudo de convergência de malha (na posição radial igual a 0 e axial igual a $3,7 \mathrm{~mm}$ do reator). A Tabela 4 mostra o número de elementos para cada malha e os valores da temperatura obtida na posição definida nas diferentes malhas. Os valores calculados para cada um dos parâmetros descritos da metodologia encontram-se na Tabela 5. Como pode ser observado foram utilizados 5 (cinco) diferentes tipos de refino para a análise da influência do refino da malha nos resultados. A malha $\mathrm{N}_{2}$ foi a utilizada no problema por apresentar $\mathrm{GCI}_{32}=0,05 \%$, resultado este, que já é satisfatório para a modelagem e por exigir menor esforço computacional na resolução do problema do reator de membrana.

Tabela 4: Número de elementos por malha e valor da temperatura calculado

\begin{tabular}{|c|c|c|c|c|c|}
\hline \multirow{2}{*}{$\begin{array}{c}\text { Número de } \\
\text { elementos }\end{array}$} & $\mathrm{N}_{1}$ & $\mathrm{~N}_{2}$ & $\mathrm{~N}_{3}$ & $\mathrm{~N}_{4}$ & $\mathrm{~N}_{5}$ \\
\cline { 2 - 7 } & 196.980 & 84.942 & 32.604 & 14.596 & 5.808 \\
\hline \multirow{2}{*}{ Temperatura $(K)$} & $\phi_{1}$ & $\phi_{2}$ & $\phi_{3}$ & $\phi_{4}$ & $\phi_{5}$ \\
\cline { 2 - 7 } & 766,94 & 767,14 & 766,65 & 764,94 & 768,51 \\
\hline
\end{tabular}

Tabela 5: Parâmetros do estudo de convergência de malha

\begin{tabular}{|c|c|c|c|c|}
\hline variável $_{i j}$ & 21 & 32 & 43 & 54 \\
\hline$r_{i j}$ & 1,3236 & 1,3760 & 1,3072 & 1,3596 \\
\hline$e_{i j}$ & 2,07 & $-0,49$ & $-1,71$ & 3,54 \\
\hline$p_{i j}$ & 2,91 & 3,93 & 3,72 & 2,50 \\
\hline$\phi_{i j}$ & 766,78 & 767,46 & 768,44 & 761,85 \\
\hline$e_{a_{i j}}$ & 0,0003 & 0,0006 & 0,0022 & 0,0047 \\
\hline$e_{e x t} i j$ & 0,0002 & 0,0004 & 0,0023 & 0,0041 \\
\hline$G C I_{i j}(\%)$ & 0,03 & 0,05 & 0,2 & 0,5 \\
\hline
\end{tabular}

As simulações realizadas através do software ANSYS CFX foram validadas pela comparação com os dados experimentais relatados em Shu et al. (1994), os valores de conversão de $\mathrm{CH}_{4} \mathrm{em}$ função da pressões de 1,5 e 2 bar apresentados por Shu et al. (1994) foram de aproximadamente 46 e $50 \%$, enquanto os obtidos através da simulação foram de 48,9 e 53,3\%. Onde foram adotados os 
parâmetros operacionais para um reator de membrana apresentados na Tabela 1, utilizando um critério de convergência para as equações de momento, calor e massa igual a $10^{-4}$. De acordo com a literatura, as variáveis que mais afetam o desempenho dos reatores de membrana são a temperatura, a pressão e a taxa de alimentação. Por este motivo, inicialmente, foi realizado o estudo da conversão de $\mathrm{CH}_{4}$ (calculada usando a vazão molar média de entrada e saída de $\mathrm{CH}_{4}$ ) e da rendimento do $\mathrm{H}_{2}$ (definido como a razão entre os números de moles de $\mathrm{H}_{2}$ permeado e $\mathrm{CH}_{4}$ alimentado) em função da pressão de operação. A Tabela 6 mostra os resultados obtidos de conversão de $\mathrm{CH}_{4}$ e rendimento de $\mathrm{H}_{2}$ em uma pressão de operação de 9, 10 e 29 bar a temperatura de 793,15 K.

Tabela 6: Conversão de $\mathrm{CH}_{4}$ e rendimento de $\mathrm{H}_{2}$ a pressão operacional de 9, 10 e 29 bar a 793,15 K

\begin{tabular}{|c|c|c|}
\hline $\mathrm{P}$ (bar) & Conversão de $\mathrm{CH}_{4}(\%)$ & Rendimento do $\mathrm{H}_{2}$ \\
\hline 9 & 56,59 & 2,09 \\
\hline 20 & 63,47 & 2,35 \\
\hline 29 & 70,88 & 2,61 \\
\hline
\end{tabular}

A Figura 2 mostra a geometria do reator de membrana com o perfil de fração molar de hidrogênio a temperatura e pressão de operação de 793,15 K e 29 bar, respectivamente. Sendo esta condição a que apresentou maior conversão e recuperação de hidrogênio.

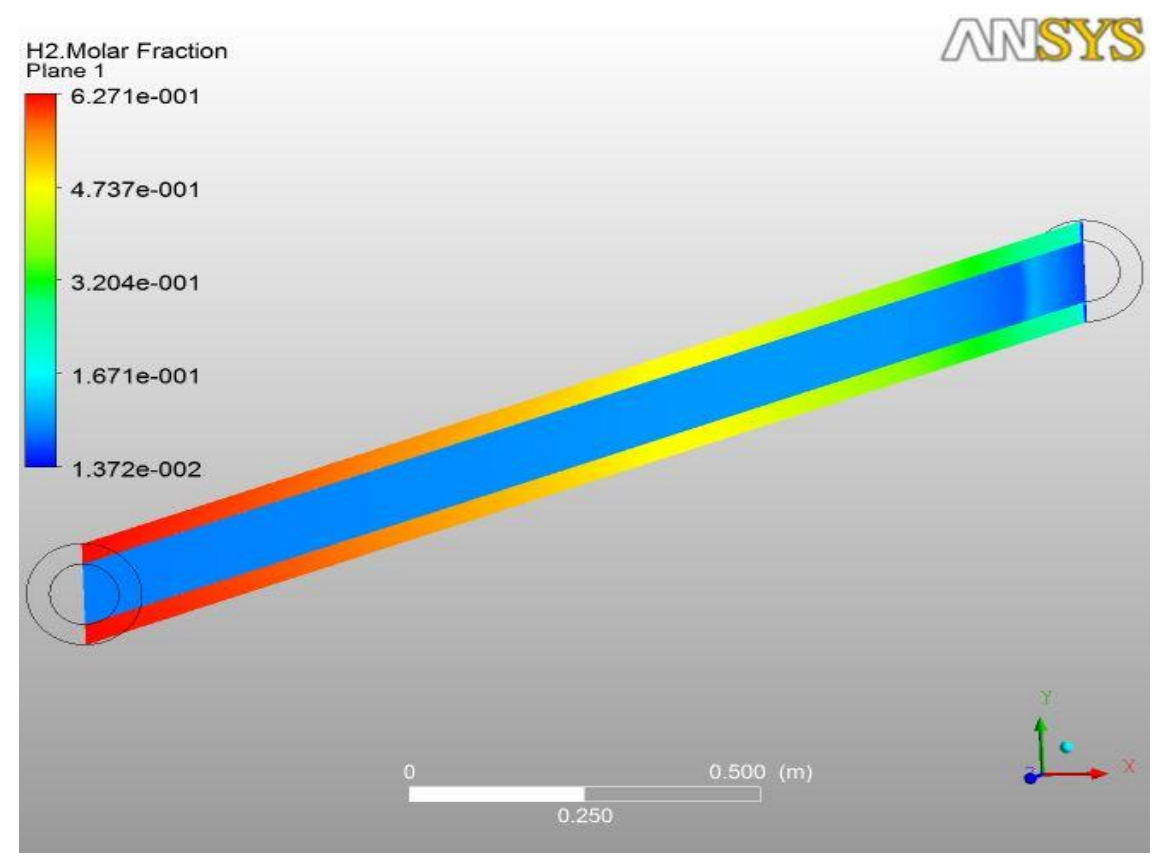

Figura 2: Perfil de fração molar de hidrogênio no reator de membrana a 29 bar e 793,15 K.

\section{CONCLUSÃO}

A convergência da malha foi alcançada utilizando a malha de 84942 elementos para o qual apresentou um índice de convergência de malha igual a 0,05\% para análise da temperatura, contudo, 

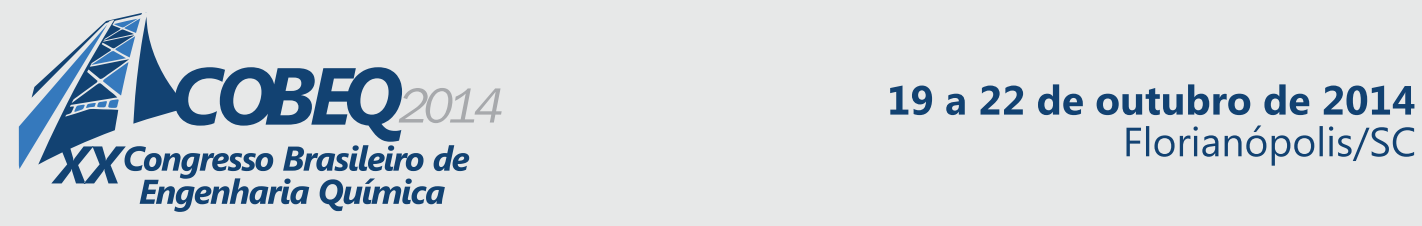

devido ao caráter oscilatório do valor de temperatura, outras posições serão analisadas para se ratificar o resultado do estudo de convergência de malha. A cinética química foi inserida como código CCL no termo de fonte para geração de massa e energia e os balanços de massa, energia e movimento obtiveram a convergência desejada. O perfil de fração molar mostrado na Figura 2 foi condizente com a literatura e os dados simulados mostraram que com o aumento pressão de operação aumentou-se o consumo do metano e a produção do hidrogênio, refletindo numa maior conversão da reação obtendose $70,88 \%$ para uma pressão de 29 bar. E por outro lado, aumentou-se também a permeação do hidrogênio através da membrana com o aumento da pressão de operação elevando dessa forma o rendimento do reator.

\section{REFERENCIAS}

ANDRADE, B. S. Estudo do Desempenho do Hidrociclone Bradley para a Separação de Biodiesel e Glicerina - Via Análise em CFD. Trabalho de Conclusão de Curso (Engenharia Química), Universidade Federal de Sergipe, São Cristóvão - Sergipe. São Cristovão, 2013;

ASME, Procedure for Estimation and Reporting of Uncertainty Due to Discretization in CFD Applications. Journal of Fluids Engineering, v. 130, p. 1-4, 2008.

BERNARDES, C. J.; Estudo da Permeação de Hidrogênio em Reator com Membrana de Paládio: Modelagem Matemática e Simulação Computacional. Dissertação Programa de Pós-Graduação em Engenharia Química, Universidade Federal de Uberlândia. Uberlândia, Minas Gerais, Brasil, 2009;

BESTETI, M. D.; SANTOS D. G. F.; CARVALHO, S. C.; ASSIS, J. A.; Avaliação Termodinâmica da Produção de Hidrogênio a Partir da Reforma a Vapor do Metano. VI Congresso Brasileiro de Engenharia Química em Iniciação Científica, Campinas, São Paulo, Brasil, 2005;

FROMENT, G. F.; XU, J. Methane Steam Reforming, Methanation and Water-Gas Shift: I. Intrinsic Kinetics. AIChE Journal, v. 35, n. 1, 1989;

MÙNERA, J., IRUSTA, S., CORNAGLIA, L., LOMBARDO, E. $\mathrm{CO}_{2}$ reforming of methane as a source of hydrogen using a membrane reactor. Applied Catalysis A: General, v. 6477, p. 1-13, 2003;

SÁ, P. F.; MATAI, P. H. L. S.; Estudo das tecnologias de produção e purificação de hidrogênio a partir de hidrocarbonetos do gás natural. In: Simpósio Internacional de Iniciação Científica da Universidade de São Paulo. São Paulo, 2004;

SHU, J.; GRANDJEAN, P. A.; KALIAGUINE, S.. Methane steam reforming in asymmetric Pd and Pd-Ag/porous SS membrane reactors. Applied Catalysis A: General, v.119, p.305-325, 1994;

SMITH, R. J. B.; MURUGANANDAM, L.; SHEKHAR, S. M.. CFD Analysis of Water Gas Shift Membrane Reactor, Chemical Engineering Research and Design, v. 89, p. 2448-2456, 2011;

SOUZA, A. C. C.; SILVEIRA, J. L.. Ensinando os Princípios Básicos da Reforma a Vapor para a Produção de Hidrogênio. Congresso Brasileiro de Ensino de Engenharia, Brasília, 2004. 